\title{
Editorial
}

\section{New Electromagnetic Methods and Applications of Antennas in Biomedicine}

\author{
Tamer Ibrahim, ${ }^{1}$ Elise Fear, ${ }^{2}$ and Stuart Crozier $^{3}$ \\ ${ }^{1}$ Departments of Radiology and Bioengineering, University of Pittsburgh, Pittsburgh, PA 15213, USA \\ ${ }^{2}$ Department of Electrical Engineering, Schulich School of Engineering, University of Calgary, AB, Canada T2N1N4 \\ ${ }^{3}$ Department of Mathematics, The University of Queensland, Queensland 4072, Australia
}

Correspondence should be addressed to Tamer Ibrahim, tsi2@pitt.edu

Received 16 July 2008; Accepted 16 July 2008

Copyright (C) 2008 Tamer Ibrahim et al. This is an open access article distributed under the Creative Commons Attribution License, which permits unrestricted use, distribution, and reproduction in any medium, provided the original work is properly cited.

Electromagnetic devices and techniques are of increasing interest and clinical utility in a variety of medical applications. Hyperthermia may be utilized to heat tissues using electromagnetic fields as part of cancer treatment. Many clinically available and emerging imaging techniques have also relied on electromagnetic concepts. Tissue-implanted devices wirelessly communicate information from the interior to the exterior of the body and vice versa. Developing and optimizing use of these technologies involves a sophisticated understanding of the interactions between electromagnetic fields and tissues. This encompasses the theoretical, computational, and experimental challenges. This Special Issue on New Electromagnetic Methods and Applications of Antennas in Biomedicine is devoted to reporting the latest research in this growing interdisciplinary field.

In this issue, the first two papers explore approaches to imaging at microwave frequencies for two different applications: stroke and breast cancer detection. The third paper examines a practical issue for breast imaging, namely, selection of an appropriate medium to immerse the antennas and breast. A study of antenna performance in immersion media is presented in the fourth paper in order to better understand issues such as variations in antenna response with immersion depth. The fifth paper presents a design for ultra-wideband antennas for microwave imaging, and analyzes the performances of these antennas in a realistic scenario. The sixth paper shifts the focus of the issue from imaging to tissue-implanted antennas. In the next three papers, the focus is shifted to magnetic resonance imaging (MRI). Antenna theory is applied to design a radiofrequency
(RF) coil for high-field MRI applications in the seventh paper. The final two papers report on electromagnetic simulation methods used in MRI. Overall, the papers in this issue represent a broad spectrum of work in new electromagnetic methods and applications of antennas in biomedicine.

We would like to thank many people whose efforts made this special issue possible. First, we thank the authors for their response to our initial call for papers. Second, we thank the team at IJAP for their ongoing support. Finally, we would like to acknowledge the significant contributions of the reviewers who provided feedback on multiple versions of the papers.

Tamer Ibrahim Elise Fear Stuart Crozier 

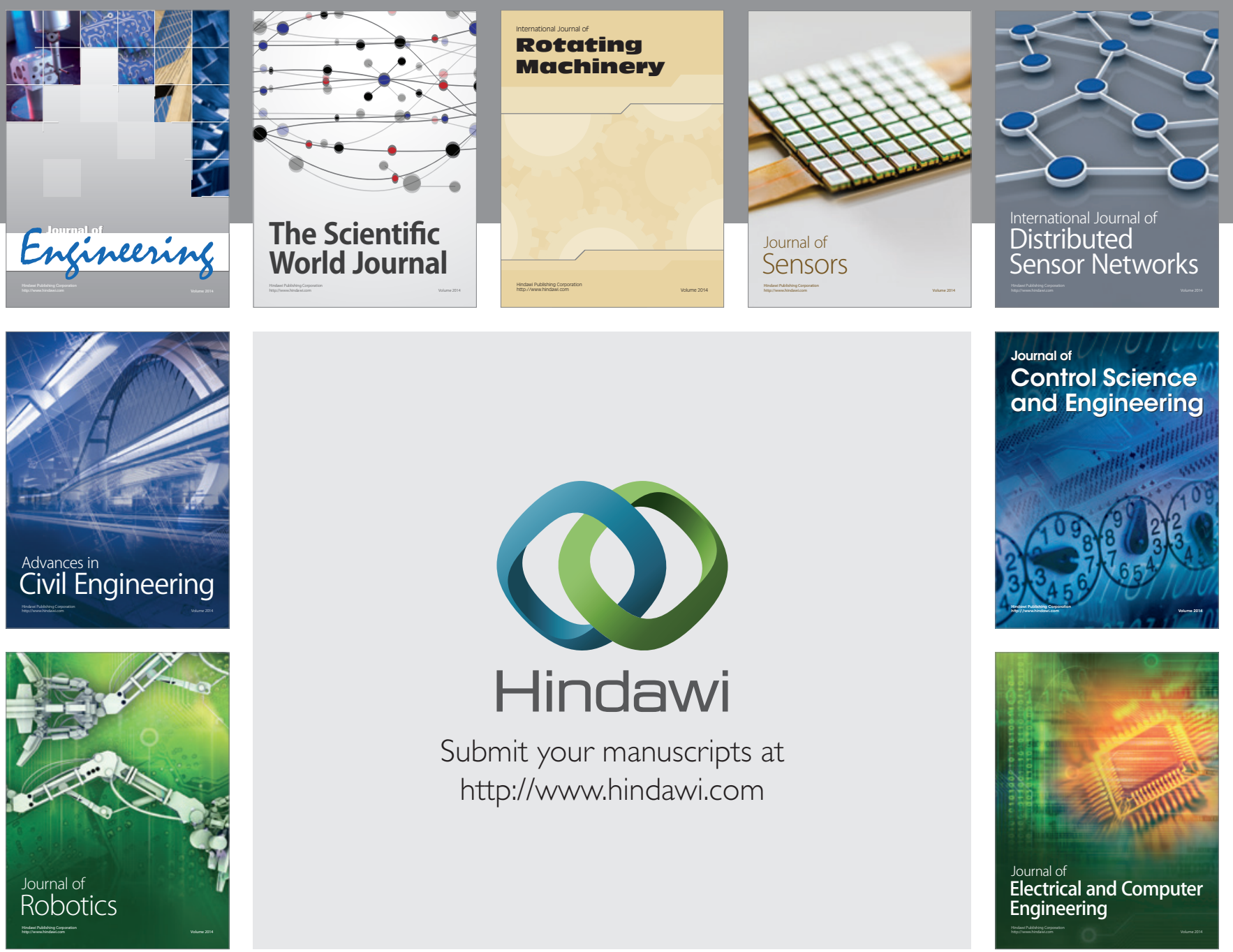

Submit your manuscripts at

http://www.hindawi.com
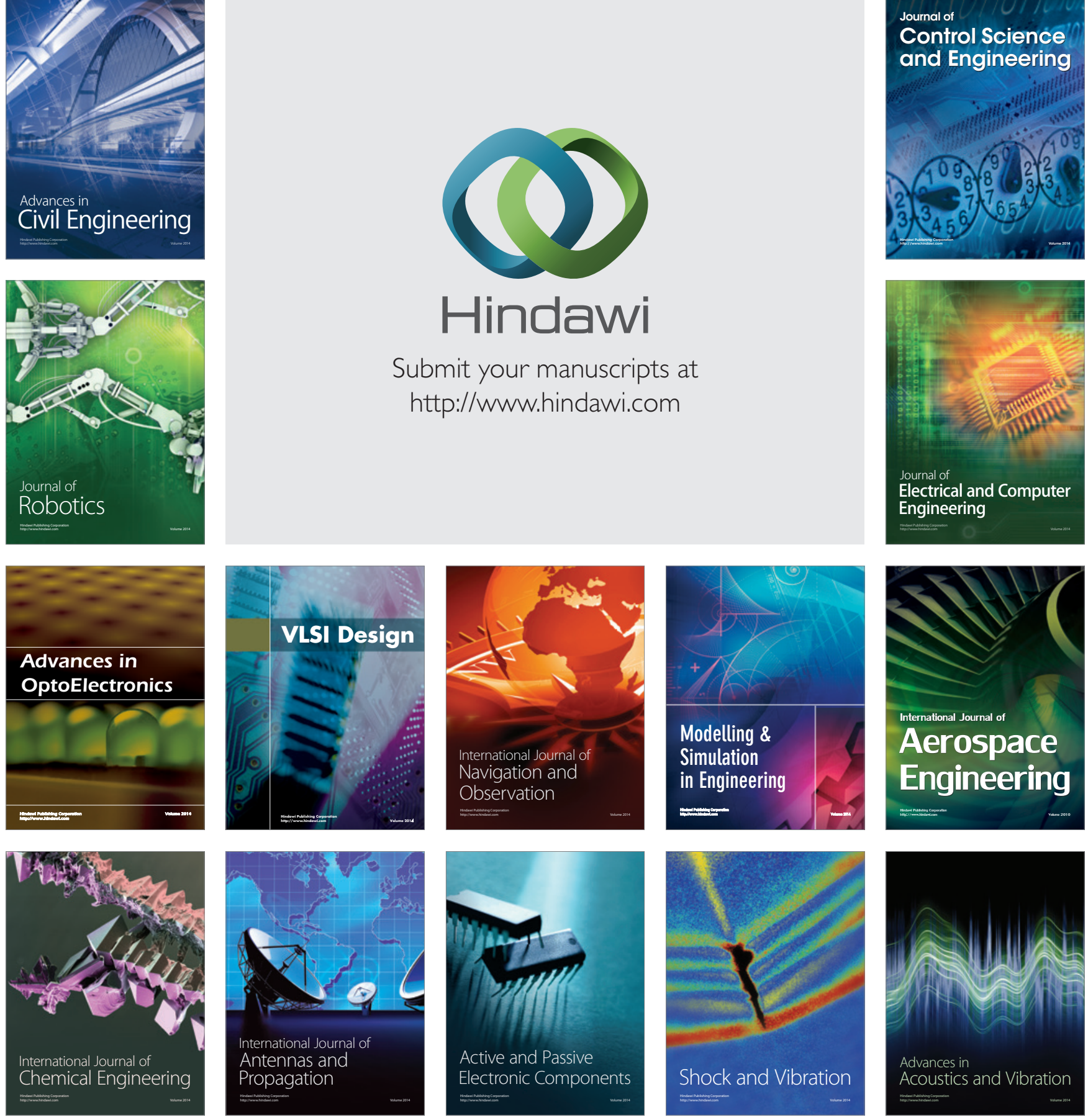